\title{
Prise en charge et stigmatisation des avortantes dans l'institution médicale : la classe des femmes sous surveillance
}

\author{
Marie Mathieu et Lucile Ruault
}

Résumé - Depuis 1975 et la délégation exclusive des avortements à la sphère médicale, l'emprise de l'État sur le travail reproductif des femmes a connu de profondes métamorphoses. L'article vise à nourrir une sociologie de l'action publique de santé, afin de saisir les recompositions de l'application de la politique de contrôle des naissances et les investissements professionnels qu'elle implique. En nous appuyant sur des matériaux variés - observation de différentes structures de prise en charge des avortements, ainsi qu'entretiens menés auprès de professionnelles de soin et de femmes ayant avorté en France -, il s'agit d'analyser les parcours institutionnels d'avortement. L'agencement des espaces, l'organisation des examens, les pratiques des professionnelles et la codification des interactions participent d'un étiquetage des usagères et d'une inertie des représentations. La stigmatisation des populations contrôlées et l'échelle de valeurs construite à propos des parcours procréatifs s'articulent avec un travail de rééducation des comportements des déviantes, alors enjointes à adhérer aux normes procréatives et contraceptives tout comme à un recours exceptionnel à l'avortement.

Politix, Volume 27 - n¹07/2014, p. 33-59 
« Des milliers de filles ont monté un escalier, frappé à une porte derrière laquelle il y avait une femme dont elles ne savaient rien, à qui elles allaient abandonner leur sexe et leur ventre. Et cette femme, la seule personne alors capable de faire passer le malheur, ouvrait la porte, en tablier et en pantoufles à pois, un torchon à la main : "C'est pour quoi, mademoiselle ?"1 "

Elles sont désormais des milliers à confier leur sexe et leur ventre à des femmes (et des hommes) pour avorter ${ }^{2}$; depuis 1975, celles-ci sont des agentes $^{3}$ de l'État en blouse blanche. L'avortement est une donnée structurelle de la vie sexuelle et reproductive des femmes ${ }^{4}$, c'est-à-dire une issue fréquente des grossesses non prévues, dévoilant différentes injonctions pesant sur elles: la planification des naissances, le désir de maternité conscientisé, une maîtrise rationnelle, anticipative et médicalisée des fertilités. Malgré les acquis législatifs et la reconfiguration des normes procréatives, la pratique de l'avorte- ment est toujours considérée comme déviante, dans la loi et son application ${ }^{5}$. Continue d'être questionné avec inquiétude le chiffre relativement stable de 200000 avortements par an en France, dont les sociologues ont pourtant mis en évidence le caractère incompressible. L'avortement est en fait pensé comme aisé- ment évitable, notamment en raison d'une large accessibilité de la contraception. Le secret continue d'entourer cet acte, qui se doit aussi d'être exceptionnel.

Si la prise en charge contemporaine de l'avortement s'inscrit dans la continuité de l'histoire du contrôle politique exercé sur les sexualités des femmes ${ }^{6}$, elle en révèle par ailleurs les nombreuses recompositions. La loi Veil signale un glissement remarquable dans la régulation étatique de ce mode de maîtrise des naissances ${ }^{7}$, qui l'éloigne du champ judiciaire et confie sa pratique et son contrôle exclusivement à la sphère médicale. Cette délégation de responsabilité a entraîné un processus de professionnalisation. Ses incidences sur le «travail re.productif $»^{8}$ des femmes et les injonctions à propos de l'ensemble des tâches que cette expression recouvre restent

\footnotetext{
${ }^{1}$ Ernaux (A.), L'événement, Paris, Gallimard, 2000, p. 77. L'auteure relate son propre avortement comme un parcours long et complexe dans le cadre de la clandestinité imposée par la répression étatique.

${ }^{2}$ Cet article est issu d'une communication présentée au congrès AFSP de juillet 2013, dans le cadre de la section thématique "Ce que le genre fait à l'État: une ethnographie du contrôle des déviances ». Nous remercions Catherine Achin, Michèle Ferrand, Sandrine Lévêque et Frédérique Matonti pour leur regard critique et leur précieux accompagnement qui ont enrichi le travail d'écriture.

${ }^{3}$ Les femmes sont majoritaires parmi les personnes impliquées dans la prise en charge des avortements en France. Voir plus loin «Une "police des femmes" par des femmes».

${ }^{4}$ Bajos (N.), Moreau (C.), Leridon (H.), Ferrand (M.), « Pourquoi le nombre d'avortements n'a-t-il pas baissé en France depuis 30 ans ? », Population et sociétés, 407, 2004.

${ }^{5}$ Divay (S.), « L'avortement : une déviance légale », Déviance et Société, 28 (2), 2004.

${ }^{6}$ Devreux (A.-M.), Ferrand-Picard (M.), « La loi sur l'avortement. Chronologie des événements et des prises de position », Revue française de sociologie, 23 (3), 1982 ; Mossuz-Lavau (J.), « La régulation des naissances : aspects politiques », Revue française de science politique, 16 (5), 1986.

${ }^{7}$ Horellou-Lafarge (C.), "Une mutation dans les dispositifs du contrôle social : le cas de l'avortement », Revue française de sociologie, 23 (3), 1982.

${ }^{8}$ Par « travail re.productif », nous entendons notamment le suivi gynécologique, le contrôle des naissances, la gestation, l'accouchement, l'élevage des enfants. Voir Vandelac (L.), « “ ... Et si le travail tombait enceinte ???”. Essai féministe sur le concept du travail », Sociologie et sociétés, 13 (2), 1981 ».
} 
cependant partiellement interrogées.

Nous proposons une réflexion sur l'encadrement, en France métropolitaine, des corps d'« avortantes » et d'avortées ${ }^{9}$, privilégiant pour cadre de pensée les théories féministes matérialistes. Pour Christine Delphy, Colette Guillaumin et Nicole-Claude Mathieu, les usages et contrôles des corps sexués sont le produit d'une division sociale du travail entre classes de sexe $\mathrm{e}^{10}$, qui consiste en une opposition/hiérarchisation entre production et reproduction; Paola Tabet a notamment souligné l'importance de l'exploitation sexuelle et du contrôle institutionnel des femmes comme « corps-outils de reproduction ». Notre analyse s'inscrit ensuite dans une sociologie de l'action publique s'attachant à saisir les pratiques, les représentations et les logiques d'ordonnancement des agents institutionnels à travers lesquelles celle-ci se fabrique et s'actualise ${ }^{11}$. Il s'agit de comprendre comment cet épisode ponctuant la trajectoire de plus d'une femme sur trois en France ${ }^{12}$ est transformé par l'institution médicale en un « événement », un processus durable et complexe, conférant à l'avortement un caractère extraordinaire. En outre, cette intervention médicale est un rappel à l'ordre des normes en matière de production d'enfants et de gestion des fertilités, hypertrophiant du reste certains mécanismes à l'œuvre dans les consultations gynécologiques de routine ${ }^{13}$.

\section{Les matériaux utilisés}

L'article est issu de deux études empiriques qui restituent les modalités d'intervention publique en se centrant sur les agentes de terrain, leur travail quotidien ${ }^{14}$, mais aussi les situations vécues des destinataires de ces politiques sanitaires. Ces recherches examinent des structures distinctes prenant en charge l'orthogénie ${ }^{15}$, points d'observation privilégiés pour comprendre l'encadrement des avortantes : principalement deux services dépendant de grands pôles hospitaliers (hors et en région parisienne) et de manière exploratoire un planning familial

\footnotetext{
${ }^{9}$ Avec le néologisme avortantes (compromis entre avortées et avorteuses), nous soulignons le caractère durable de l'épisode en train d'être observé et désignons une catégorie particulière de patientes, tout en tentant de nuancer le présupposé de passivité des femmes véhiculé par le terme avortées. « Avortée »-plus couramment usité - évoquera ici le caractère passé de l'épisode au moment de l'enquête.

${ }^{10}$ Raisonner en termes de « classe de sexe » permet de souligner qu'hommes et femmes sont des constructions sociales. Il s'agit dès lors d'analyser les modes de constitution de la dichotomie sexuée : les groupes de sexe sont construits à travers des rapports de domination, d'exploitation et de contrôle, autour d'intérêts antagoniques. Ce raisonnement réfute toute conception naturaliste des catégories et considère le genre comme préexistant au sexe.

${ }^{11}$ Lipsky (M.), Street Level Bureaucracy. Dilemmas of the Individual in Public Services, New York, Russell Sage Foundation, 1980.

${ }^{12}$ Bajos (N.), Prioux (F.), Moreau (C.), "Increase of repeat abortion in France: From contraceptive issues to postponement of childbearing age", Revue d'Épidémiologie et de Santé Publique, 61 (4), 2013.

${ }^{13}$ Guyard (L.),La médicalisation contemporaine du corps féminin :le cas de la consultation gynécologique, thèse pour le doctorat en sociologie, Université Paris X, Nanterre, 2008 ; Ruault (L.), À la santé de ces dames ! Penser politiquement un suivi médical : gynécologie et contrôle des corps de femmes, mémoire de sociologie politique, Université Lille 2,2011.

${ }^{14}$ Dubois (V.), «Ethnographier l'action publique. Les transformations de l'État social au prisme de l'enquête de terrain », Gouvernement et action publique, 1 (1), 2012.

${ }^{15}$ Ensemble des mesures de planification des naissances, dont la réalisation des avortements.
} 
(association «semi-médicalisée » assurant des entretiens pré-/post-IVG et des avortements par médication ${ }^{16}$ ), ainsi qu'une structure médicalisée d'une maternité. Ont été réalisés quinze entretiens avec des gynécologues, sept avec des conseillères de planification familiale, médecins généralistes, infirmières et des observations directes d'interactions d'avortantes avec sept médecins et un conseiller conjugal. Des lieux plus «privés », au double sens du terme, ont également été enquêtés à travers trente entretiens menés entre 2007 et 2014 avec des avortées : des cabinets médicaux en libéral, des cliniques et laboratoires privés, mais aussi les domiciles des femmes lorsqu'elles avortent par médication chez elles. Cette combinaison originale permet de saisir, en actes, la vigilance publique s'exerçant sur la gestion des fertilités des femmes et l'usage de leur corps. En ethnographiant les discours et les pratiques des « soignantes », on peut rendre compte des modalités d'application des politiques publiques en ce domaine et saisir par une approche micro les catégories d'entendement de ces groupes professionnels, mais aussi les tactiques et logiques identitaires à l'œuvre dans les interactions, dont l'interprétation nécessite, en miroir, les discours ${ }^{17}$ d'avortées.

De l'application des règlements découle une diversité de pratiques internes aux structures. La pluralité des positions dépend également de la hiérarchie et de la compétition dans la définition des domaines de compétences entre les différents corps professionnels - infirmières, aides-soignantes, sagesfemmes, gynécologues, généralistes et conseillères conjugales ; le matériau mobilisé ne permet pas d'analyser ces nuances, qui ne sont pas l'objet direct de cet article. Et, au-delà des enjeux identitaires des intervenantes (dont les conceptions de leur métier) et de leur appréciation différenciée de l'ordre social, ressort un troublant accord sur les bienfaits de l'encadrement institutionnel des avortantes. Les professionnelles de soins spécialisées dans la santé sexuelle des femmes, dépositaires - symboliquement du moins - des luttes féministes pour l'avortement libre et gratuit, sont ainsi porteuses d'une ambivalence qui s'apparente à celle de «nombre de porte-parole en faveur de l'avortement [...] d'un côté, elles s'insurgent contre toute intervention qui aurait pour but ou pour effet de "culpabiliser" les femmes qui avortent, et, de l'autre, rappellent avec insistance qu'il serait contraire à la réalité, et insultant pour les femmes qui l'accomplissent, de traiter cet acte "à la légère", de le "banaliser" $\gg{ }^{18}$. On ne peut donc négliger les tensions contradictoires, voire les conflits intérieurs que suscitent les contraintes de rôle chez certaines agentes, dont font peut-être partie les médecins pionniers/ères des batailles pour le droit à l'avortement et fondateurs/trices de CIVG (Centre d'interruption volontaire de grossesse) au lendemain de la loi.

\footnotetext{
${ }^{16}$ En France, l'avortement médicamenteux correspond à une combinaison de mifépristone (RU 486) et misoprostol, dont les prises sont espacées de 36 à 48 heures. L'autre méthode usitée est l'avortement par aspiration.

17 Dubois (V.), «Le rapport des assistés aux organismes sociaux: domination, tactiques et logiques identitaires », Pyramides, 7,2003.

${ }^{18}$ Boltanski (L.), La condition fotale. Une sociologie de l'engendrement et de l'avortement, Paris, Gallimard, 2004, p. 280-281.
} 
Nous montrerons dans un premier temps comment le traitement des avortantes par les institutions médicales souligne les manquements des femmes aux normes sociales en vigueur, notamment celle de la maternité «biologique ${ }^{19}$. Pour rendre compte des dispositifs à l'œuvre, l'approche ethnographique sera attentive aux lieux traversés par les femmes en demande d'avortement et, au cœur de leur cheminement, aux cabinets médicaux où l'on a pu observer des interactions avec des professionnelles de soins, en l'occurrence majoritairement des femmes. Nous centrerons ensuite la réflexion sur le stigmate avortement tel qu'il est construit en ces structures. Il est un outil central de la (ré)éducation des avortantes par des agentes de l'État, à la fois éducatrices et surveillantes des pratiques légitimes en matière de contrôle des fertilités. Nous verrons dans cette seconde partie que l'application différenciée des dispositifs d'encadrement s'appuie notamment sur une échelle de déviances, signifiante pour la définition de la classe des femmes.

\section{Signifier la déviance au féminin}

\section{Sur les chemins de l'avortement, une maternité heureuse qui s'expose}

Les cadres spatiaux de l'avortement se réfèrent sans cesse à ce qui est précisément construit comme son inverse. À commencer par les intitulés des services hospitaliers où s'effectuent les avortements et consultations pré-/post-IVG : le champ lexical de l'enfantement (mère, nouveau-né, maternité, etc.) suggère que l'on vient avorter dans un lieu valorisant la fonction maternelle. Dans les structures enquêtées, l'expression "planification conjugale et familiale » est omniprésente : inscrite au sol, sur la façade de l'hôpital, à la porte de bureau de la conseillère, sur les brochures fournies. Ces termes renvoient les avortantes à une injonction au «faire famille» pensée comme tributaire du «faire couple». La «planification» implique une éthique de la gestion responsable des fertilités par les femmes ainsi que la survenue future d'une naissance programmée, traduction matérielle de l'opposition maternité/avortement conceptualisée par la $\operatorname{loi}^{20}$. D'ailleurs, la substitution sémantique de l'avortement par l'équivoque expression interruption de grossesse suggère non pas l'arrêt, mais une pause pour une reprise ultérieure du processus ${ }^{21}$.

Dans les centres hospitaliers enquêtés, le service d'IVG partage un étage avec les suivis obstétriques. Il n'est pas rare que les avortantes soient

\footnotetext{
${ }^{19}$ Mathieu (N.-C.), « Paternité biologique, maternité sociale... », in Michel (A.), dir., Femmes, sexisme et sociétés, Paris, Presses universitaires de France, 1977.

${ }^{20}$ Dans la loi, l'IVG apparaît dans une section du Code de la Santé Publique intitulée « Santé de la famille, de la mère et de l'enfant », dans le Livre II, c'est-à-dire juste après le Livre I dédié à la Protection et promotion de la santé maternelle et infantile.

${ }^{21}$ Malenfant (A.), La nouvelle norme procréative. Regard sur les contradictions du projet parental vécues dans le cas de grossesses non prévues, mémoire pour la maîtrise de sociologie, 2011, p. 24-25.
} 
confrontées à un attroupement de couples de futurs parents venus visiter les services. La maternité s'impose alors à elles de différentes manières :

N (31 ans, chômeuse, fort capital culturel ; ayant avorté en clinique) : « Ça a été complètement horrible, une chambre glauque... c'était dans une maternité en même temps, donc j'entendais les bébés pleurer dans la chambre d'à côté. »

J (29 ans, emploi précaire, fort capital culturel ; ayant avorté à l'hôpital) : «C'est un peu paradoxal aussi : à la fois c'est la maternité et à la fois c'est aussi (elle rit) où tu peux faire un IVG. J'ai juste trouvé qu'ils étaient vraiment très accueil- lants et je me souviens que je me disais que si je veux avoir des enfants je vais quand même accoucher ici. Maintenant, non, je pense pas que je vais le faire, car l'endroit veut dire autre chose pour moi maintenant. »

Le rassemblement dans une même enceinte des divers traitements possibles des grossesses - «80\% de l'activité d'IVG est intégrée à un service de gynécologie-obstétrique en $2007 »^{22}$ - répond autant à une logique gynocentrée qu'à des contraintes organisationnelles. Toutefois, il n'a de sens que rapporté aux schèmes de perception des parcours procréatifs de femmes : accoucher et avorter constituent socialement des actes médicaux non comparables et hiérarchisés, de «valeur » inégale, de sorte que la proximité des deux services interroge, voire dérange les avortantes.

Du reste, cette contiguïté apparente va de pair avec des inégalités de traitement: par contraste avec l'accouchement, l'accompagnement personnalisé de l'avortante est pour partie refusé :

L (30 ans, un enfant, thésarde au chômage) : « Ce qui est con... mais lui (son conjoint) il acceptait de venir avec moi, 'fin, je voulais qu'on le vive ensemble, je sais pas pourquoi, mais j'avais besoin de sa présence, et puis lui était pas réticent à l'idée de m'accompagner. Et des gens nous avaient dit que c'était possible, à la maternité. Et en fait, quand ils m'ont préparée pour aller au bloc et qu'il a voulu monter dans l'ascenseur, ils m'ont dit "Non, non, mais... (elle rit) Hors de question. Vous y allez toute seule", et là, panique à bord. J'étais avec deux médecins, enfin, sur le vif, qu'on me dise qu'il devait attendre en bas, alors que jusqu'à ce moment-là on pensait qu'on allait être à deux... Han! je me suis sentie toute seule dans cet ascenseur [...] l'impression d'être prise dans un étau en métal quoi, tu vois. Avec deux médecins en blouse blanche. Ah! (elle rit) un peu la panique, l'image violente. »

Repoussé.e.s vers la salle d'attente, et ce " pour préserver l'intimité » des avortantes, ces accompagnant.e.s s'effacent devant l'interaction individualisée avec des expertes de santé. L'avortement semble ainsi devoir

\footnotetext{
${ }^{22}$ DREES, « Les établissements et les professionnels réalisant des IVG », Études et résultats, 712, 2009.
} 
être vécu retirée dans un bloc, isolée des proches. Les protocoles produisent de plus une inégale répartition sexuée du travail re.productif, puisque la présence des partenaires sexuels lors des actes d'avortement est refusée ${ }^{23}$. De même, dans un des centres de planification observés, les informations s'adressent exclusivement aux femmes, à l'exception d'un feuillet sur un présentoir à l'entrée du service "L'IVG : une histoire d'hommes aussi... ». Plus généralement, les rares références masculines ne contrebalancent pas l'asymétrie de traitement réservé aux deux sexes dans les espaces du parcours-type des avortantes.

Les lieux observés, services hospitaliers, mais aussi cabinets privés - qui accueillent une patientèle plus homogène et dotée en capitaux économiques et scolaires -, ont en commun de mettre en scène la maternité auprès de femmes refusant justement l'enfantement. La décoration et l'agencement des espaces reflètent le fort investissement dont fait l'objet le corps reproducteur féminin, et exposent un modèle situé de maternité. Parmi les messages entourant les avortantes, on remarque l'abondance des normes définissant une maternité réjouissante, saine et attentive à la santé du futur être. Dans les salles d'attente, les posters des campagnes de prévention préconisant «Zéro alcool pendant la grossesse » et la vaccination systématique - « Transmettezlui votre amour, pas la coqueluche»; "protégez votre bébé»-, les images de foetus, d'allaitements sereins, de ventres ronds sur lesquels se pose une main bienveillante, ainsi que les magazines dédiés aux mères (et parents), glorifient l'amour maternel et l'enfant à venir.
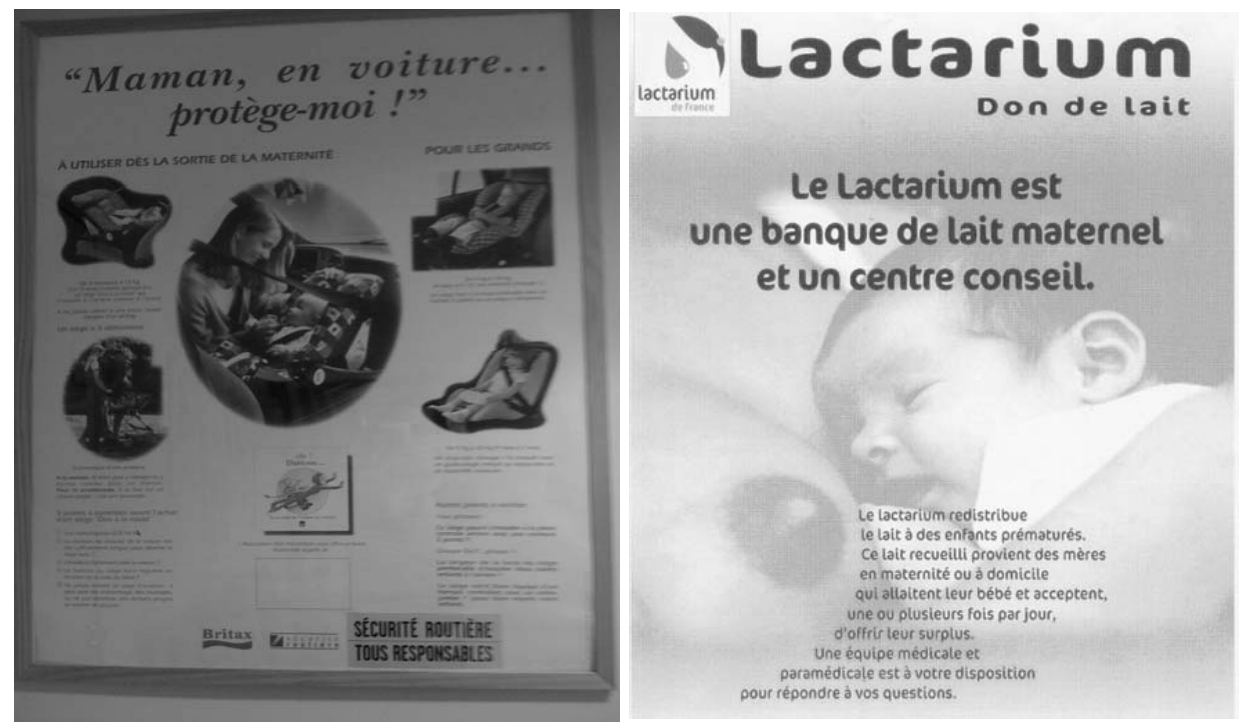

${ }^{23}$ Une récente recherche souligne déjà la difficile implication des hommes dans la gestion des fertilités: Ancic, Enquête sur le rapport des hommes à l'IVG. Rapport de l'enquête quantitative et qualitative, année 2010-2011. 
Les protagonistes sont des femmes alliant capital esthétique et attributs maternels - blanches, minces, souriantes, dévouées à l'enfant -, représentées comme responsables, prudentes et très souvent seules. Ces étapes valorisées de la vie féminine - grossesse, accouchement, allaitement au sein et élevage $^{24}$ des enfants - sont autant de rappels à l'ordre visuels auprès de celles transgressant ce modèle, autrement dit les déviantes de la maternité. Ils peuvent exercer une violence symbolique sur celles qui souhaitent avorter malgré leur intériorisation plus ou moins forte de la norme de la maternité biologique, contrariée par un contexte social perçu comme inapproprié pour la naissance d'un enfant. Cela participe de la dépréciation des dominées, qui peuvent admettre la légitimité de cette échelle des valeurs. C'est le cas de $\mathrm{N}$ qui évoquait plus haut le malaise créé par les cris de nouveau-nés :

«J'ai quand même fait trois avortements et $\mathrm{j}$ 'ai pris conscience que pour les trois grossesses j'avais pas DU TOUT envie d'avorter, que mon corps, mon cœur, mon âme, tout me poussait à me dire "c'est super, c'est magnifique, vive la vie", et que c'est ma tête, heureusement un peu sur mes épaules, qui m'a dit "non, c'est juste pas possible”. »

Parmi les images qui ponctuent le parcours d'avortement, la plus marquante est peut-être l'échographie ${ }^{25}$, pièce du dossier médical: les usagères ont bien souvent en possession cette empreinte graphique, et la conservent parfois jusqu'à l'exposer à la sociologue en entretien. En ajoutant à un état corporel - qu'elles se doivent légalement d'éprouver lors du délai de réflexion (d'une semaine) imposé - une représentation concrète, cet examen renvoie subjectivement les avortantes à un autre état, s'apparentant à une procréation souhaitée, et au suivi d'une maternité en train de se construire $^{26}$. Il s'agit là d'une incitation à se penser comme mère potentielle et à considérer toute conception comme un être en devenir.

Dans ces espaces, la faculté procréative est construite comme pan positif de l'encadrement médical de la classe des femmes. Cette toile de fond participe ainsi de la construction d'un vécu dramatique de l'avortement ${ }^{27}$. C'est précisément parce qu'il est un «refus culturel d'un processus biologique entamé» ${ }^{28}$ que l'avortement ne peut se comprendre indépendamment de l'assignation des femmes à l'enfantement, et de l'approche «biocentriste» de ce groupe de sexe. Dans l'enceinte médicale, les avortantes sont renvoyées de manière redondante au rôle de mère

\footnotetext{
${ }^{24}$ Désigne l'ensemble des soins et attentions matérielles et affectives fournis par des adultes aux enfants. Cette fonction est traditionnellement déléguée aux femmes dans la division sexuée du travail domestique prévalant au sein des structures familiales. Ces tâches non rémunérées sont analysées par les féministes matérialistes comme partie prenante de l'exploitation du travail des femmes par la classe des hommes.

${ }^{25}$ Le délai d'avortement justifie actuellement le recours à l'échographie pour apposer un chiffre, en nombre de semaines, à l'état de grossesse.

${ }^{26}$ Fellous (M.), « La révolution échographique », Sociologie du travail, 30 (2), 1988, p. 311-321.

${ }^{27}$ Lambert (S.), L'injonction au drame, représentations et expériences vécues de l'interruption volontaire de grossesse, mémoire pour le master 2 de sociologie, Université de Poitiers, 2012.

${ }_{28}$ Mathieu (N.-C.), L'Anatomie politique. Catégorisations et idéologies du sexe, Paris, iXe, 2013 [1991], p. 63.
} 
potentielle, soit au versant heureux d'une expérience qu'elles vivent souvent, selon nos observations, dans la solitude, la patience et le silence.

\section{Une " police des femmes" par des femmes}

Ces arènes d'encadrement médicalisé sont, de manière flagrante, des entre-soi féminins. Aux avortantes s'ajoutent le plus souvent des accompagnantEs, bien que cantonnées aux salles d'attente. Cette absence masculine s'inscrit pleinement dans le suivi médical plus général des pratiques sexuelles et reproductives, surveillées sur les seuls corps des femmes, qui servent volontiers d'outils pour en exempter les hommes ${ }^{29}$. Même lorsque «le caractère concret de la fécondation ne permet plus de délier complètement les responsabilités $»^{30}$, l'expérience médicalisée d'avortement est déléguée pour l'essentiel aux femmes. On peut interpréter cette enclave comme la reconnaissance de l'autonomie décisionnelle des femmes portée par la loi, et dont l'institution médicale assure le respect. Mais cette ségrégation spatiale périodique est aussi et surtout partie prenante de $«$ l'arrangement des sexes $»^{31}$, accomplissant et confortant la « différence essentielle» et l'infériorisation des femmes tout en reproduisant le lien traditionnel entre procréation et féminité.

Au-delà des populations-cibles, c'est la maîtrise procréative dans son ensemble qui est souvent conduite entre femmes. D'abord, les figures institutionnelles encadrant les avortements sont majoritairement issues de la même classe de sexe; au niveau national, les femmes (sages-femmes, gynécologues et généralistes) représentent officiellement $58 \%$ du personnel médical ${ }^{32}$ impliqué dans la prise en charge des IVG - statistique minorée ${ }^{33}$, et ne tenant pas compte de l'implication des infirmières, conseillères conjugales et assistantes sociales. De plus, la surreprésentation féminine dans les métiers des soins et du secrétariat, qui occupent un rôle important dans les parcours d'avortement, implique que l'accueil, les démarches administratives, les prises de sang et de médicaments, les soins de confort et de dialogue soient assurés par des femmes. En outre, n'oublions pas que l'autre pan de la maîtrise de la procréation, la contraception, est d'abord encadré en

\footnotetext{
${ }^{29}$ Les parcours de procréation médicalement assistée focalisent aussi les interventions et traitements lourds sur les femmes. Voir Akrich (M.), Laborie(F.), dir., Cahiers du genre, 25, De la contraception à l'enfantement : l'offre technologique en question, Paris, L’Harmattan, 1999 ; Löwy (I.), Rozée Gomez (V.), Tain (L.), dir., Cahiers du genre, 56, Biotechnologies ettravailreproductif. Uneperspectivetransnationale, Paris, L'Harmattan,2014.

${ }^{30}$ Pache (S.), « Au nom de la santé. La légalisation de la contraception et de l'avortement», communication au colloque «Comment l'État fait-il notre lit? La régulation des sexualités en Europe », Université libre de Bruxelles, mars 2010.

${ }^{31}$ Goffman (E.), L'arrangement des sexes, Paris, La Dispute, 2002 [1 $1^{\mathrm{re}}$ éd. am. 1977], p. 79-82.

${ }^{32}$ DREES, « Les établissements et les professionnels réalisant des IVG », art. cit., p. 6.

${ }^{33}$ Après vérification auprès de la DREES, il s'avère que l'enquête par questionnaire ignore dans quelle mesure les sages-femmes agissant sous délégation de médecin ont noté les références du médecin au lieu des leurs pour chacune des IVG enquêtées. Sachant que 97,6\% (DREES, 2014) des sages-femmes sont des femmes, les $58 \%$ de répondantes de l'enquête de 2007 sont certainement à redresser.
} 


\section{gynécologie par les femmes ${ }^{34}$.}

Sur nos terrains, cette division socio-sexuée du travail en santé sexuelle et reproductive était d'autant plus marquée que les gynécologues médicales assuraient l'essentiel des consultations pré- et post-IVG ainsi que des réalisations d'IVG. Dans les services gynécologiques enquêtés, nous avons donc principalement observé l'assignation des praticiennEs au versant réputé négatif de la fonction reproductive des femmes. Cette donnée ne peut être évacuée dans l'analyse des négociations, car le contrôle social exercé entre femmes recouvre sans doute des dynamiques particulières ${ }^{35}$. Dans ces interactions, au-delà de la frontière entre médecin et non-médecin, on peut en effet interroger le partage d'une expérience commune, issue d'une inscription particulière dans les rapports de domination. L'appartenance à une classe de sexe que les structures en charge de l'avortement signifient aux usagères s'étend-elle jusqu'aux professionnelles médicales ?

Une étape importante du travail institutionnel d'assignation symbolique des avortantes consiste en la mise en place d'une "police des récits $»^{36}$, enjoignant les femmes à dire les circonstances de survenue de la grossesse et les motifs de son refus. Depuis la disparition en 2001 de l'entretien obligatoire avec la conseillère conjugale ou l'assistante sociale, ce recueil de la parole est incorporé dans une certaine mesure aux consultations médicales. $\mathrm{P}$, infirmière ayant travaillé dans le centre de planification d'un hôpital de banlieue parisienne, rend compte des difficultés rencontrées dans la mise en place de groupes de parole pour les avortantes :

« On aurait bien aimé, et puis en fait il y a eu des résistances de part et d'autre, donc on a presque... Parce qu'après il y a le conflit de savoir qui va les animer ? [...] C'est vrai que des fois, il y a des petites dissensions dans l'équipe à ce niveau-là, que chacun trouve sa place. "Oui, mais là, tu me piques mon travail" [...] Voilà, on avait du mal chacun à trouver sa place. [...] Les conseillères et nous les médicales, je pense y'a eu un moment elles ont vu que moi je piquais, voilà, on essayait chacun d'être à sa place et tout ça. »

La modification de la loi semble avoir nourri un brouillage des frontières entre les domaines d'action des différentes professionnelles, allant dans le sens d'un élargissement de la fonction des médecins à la prise en charge psychologique et morale. Ce glissement du travail de production de la parole assurerait la pérennisation de ce qu'Anne-Marie Devreux nomme «l'effet inductif de l'institution », soit la production structurelle d'une « nécessité de

\footnotetext{
${ }^{34}$ La gynécologie médicale est composée à $70,3 \%$ de femmes, contre 39,6\% en obstétrique. Sicart (D.), « Les médecins au 1 ${ }^{\mathrm{er}}$ janvier 2012 », DREES, Document de travail, Série statistiques, 167, 2012.

${ }^{35} \mathrm{Cf}$. notamment Cardi (C.), « Le contrôle social réservé aux femmes : entre prison, justice et travail social », Déviance et Société, 31 (1), 2007.

${ }^{36}$ Memmi (D.), Faire vivre et laisser mourir. Le gouvernement contemporain de la naissance et de la mort, Paris, La Découverte, 2003.
} 
parler $\aleph^{37}$ pour les avortantes. En explorant les consultations médicales qui jalonnent les parcours d'avortement, nous pouvons repérer cette obligation et les normes sous-jacentes qui cadrent les discours.

De façon générale, l'obligation officieuse de rendre des comptes en matière de procréation n'advient qu'en situation de demande d'avortement ou de difficulté à concevoir, c'est-à-dire quand pouvoir et vouloir ne se rejoignent pas. Cette asymétrie dans le dispositif de recueil de la parole entre les avortements et les grossesses désirées est un mécanisme fabriquant la « déviance »: les avortantes sont tenues de justifier un manque de maîtrise rationnelle et efficace de leurs fertilités alors qu'elles manifestent «après coup » le rejet d'une potentielle maternité.

Les croyances des professionnelles que rencontrent les usagères au cours des différentes étapes rendues nécessaires pour avorter peuvent percer à travers leur attitude :

I (28 ans, détentrice d'un DEA, journaliste pigiste) : «J'ai mal vécu la prise de sang au laboratoire. J'ai eu une réflexion désagréable d'une infirmière. Alors, je sais qu'elle, peut-être qu'elle voulait être sympa en me demandant "Oh, c'est pour une grossesse alors, tout ça ?". Et moi, j'ai été assez catégorique, je lui dis "Oui, enfin non. Je vais avorter". Et bon, elle a eu un regard désagréable, elle m'a fait la morale. Donc ça, j'ai pas du tout apprécié, c'était pas son rôle. Voilà, c'est pas ma mère, c'est pas quelqu'un de ma famille. C'est pas mon médecin traitant. »

Dans cette interaction telle qu'elle est vécue par I, l'infirmière insinue une perception positive de la grossesse destinée à créer un nouvel être et, à l'inverse, négative de celle arrêtée. I poursuit : «Avec la grossesse et donc l'avortement, la ligne entre le médecin neutre, professionnel, et puis les sentiments de l'homme ou de la femme qu'il y a derrière, cette ligne-là est souvent franchie. » Ce témoignage sur le pouvoir moral des professionnelles invite à la compréhension des processus de régulation étatique de la (non)reproduction par l'exploration d'un pan particulier, celui d'une police des femmes sur les femmes.

$\mathrm{Au}$ cours des séquences observées - consultations pré- et post-IVG, échographies -, les gynécologues-femmes, contrairement à leurs propres présupposés sur leurs incarnations «particulières » de l'autorité médicale et leur empathie accrue, ne mettent pas (ou peu) en avant leur expérience sociale de femmes. L'attitude prévalant dans les interactions avec les avortantes consiste à valoriser leur rôle d'experte face à une profane, et non leur commune appartenance à une classe de sexe (dont leur potentielle expérience d'avortement). Cela peut prendre la forme de remontrances

\footnotetext{
${ }^{37}$ Devreux (A.-M.), « De la dissuasion à la normalisation. Le rôle des conseillères dans l'entretien pré- IVG », Revue française de sociologie, 23,1982.
} 
personnalisées : "Une infirmière doit savoir qu'il y a $10 \%$ de grossesses avec préservatif! Même quand il ne craque pas et qu'il est mis à chaque fois », remarque, sur un ton las et calme, une gynécologue auprès d'une avortante de 37 ans, infirmière. Cette négation des compétences d'une professionnelle de soins, qui se traduit plus généralement dans d'autres interactions par une mise à distance des savoirs profanes, est d'autant plus remarquable que l'avortement est un acte prescrit par l'usagère, non malade, face à un.e médecin exécutant.

Historiquement, la médicalisation de l'avortement a opéré un glissement $\mathrm{du}$ comportement des «avorteuses». Quand les mouvements pour l'avortement libre des années 1970 promouvaient une conscience de classe de sexe, les praticiennes ont plutôt intégré une éthique de la "neutralité » médicale qui prime sur leur identité de femme. Les premiers, partisans d'une prise en charge collective, ont laissé place dans l'institution médicale à des relations de face-à-face qui exacerbent la dimension de contrôle. Ces mécanismes ont pour effet d'accentuer la distance sociale entre professionnelle et profane tout en affirmant une inégalité de maitrise; ils participent de la production d'une altérité qui freine la solidarité et l'alignement d'intérêt entre femmes. Les avortantes se perçoivent donc en « contacts mixtes $»^{38}$ avec des «normales», soit des femmes incarnant $a$ priori des normes corporelles dominantes. Celles-ci apparaissent d'abord comme des "modèles", des professionnelles de santé "pures», porteuses des normes et garantes de leur respect.

M (24 ans, future avocate) : «La première [gynécologue] que j'ai eue, elle, c'est une grosse conne. [...] Elle est vachement dans le jugement en fait [...] C'était celle de ma mère, mais je l'ai jamais trop vraiment aimée, parce que déjà, je fais ce que je veux (rires) de mon corps, et puis, je sais pas... J'ai eu mes premiers rapports, c'était à 18 ans. Donc faut pas déconner. C'est pas comme si j'avais fait n'importe quoi. Et puis, là en l'occurrence, si je suis tombée enceinte, c'était un accident. Je peux pas prendre la pilule. Elle avait rien à dire, elle avait pas à me juger... Mais j'aimais déjà pas cette femme à la base. »

Certes, les avortantes peuvent parfois contrôler l'information et mobiliser des stratégies pour éviter le risque de se voir "moralisées» - cette disposition à décrypter l'interaction médicale étant inégalement distribuée. Par leur socialisation, elles ont appris et intégré la prescription sociale de concordance entre gestion de la fertilité et projet parental, ainsi que le pan honteux que représente l'avortement dans le parcours procréatif menant à la «belle» maternité. C'est ainsi que certaines avortantes dissimulent lors des interactions médicales le fait de ne pas avoir utilisé de contraceptif. Si elles peuvent adhérer à cette échelle de valeurs, elle est fortement réactivée au

\footnotetext{
${ }^{38}$ Goffman (E.), Stigmate. Les usages sociaux des handicaps, Paris, Minuit, 2010 [1 ${ }^{\text {re }}$ éd.1975], p. 23.
} 
contact de professionnelles de santé, qui contribuent à l'incorporation de ces normes.

\section{Garder à vue les dissidentes : une formation accélérée à une sexualité responsable}

\section{Signifier la déviance : la construction d'un événement exceptionnel}

Dans la loi, l'avortement n'est pas considéré comme un moyen ordinaire pour les femmes de maîtriser leur fertilité ${ }^{39}$; il est conçu comme un geste non consensuel ${ }^{40}$ d'arrêt de la vie, dont il reviendrait à un ensemble de professions désignées par l'État d'assurer la surveillance. L'institutionnalisation de l'avortement a été construite comme dépendante de l'accessibilité à une contraception médicale - les deux lois Veil, sur une séquence historique courte ${ }^{41}-$, assimilant d'emblée l'avortement à la situation à éviter à tout prix, opposée à la contraception - recours « évident», moindrement invasif, et premier (en amont du rapport sexuel). Cette dialectique s'est alors traduite dans le droit par un accès simplifié et plus large à une contraception « préventive » qu'à l'avortement.

La pratique abortive dans le champ médical fait partie des actes réputés techniquement médiocres, peu rémunérateurs et disqualifiants. Ce «sale boulot» demeure une tâche subalterne dont l'évitement est facilité par la clause de conscience obtenue par le corps médical lors du vote de la loi Veil. La prise en charge des avortements est encore relativement complexe en France - comparée, par exemple, au Québec -, signifiant par là même la déviance qu'elle représente par rapport à la norme d'une gestion rationnelle, anticipée et médicalisée des fertilités des femmes. Reflet de la lourdeur administrative du système de santé français, le chemin effectué par certaines femmes pour obtenir un avortement peut ressembler à un parcours de la combattante, d'autant que le démantèlement des structures IVG en rend l'accès difficile.

Le Code de la Santé publique impose un trajet dense et codifié d'interactions et interventions médicales, un cadre à application variable. Bien souvent, les structures et professionnelles en charge des avortements les traduisent et se les approprient dans le sens d'une surenchère, une multiplication dans l'espace et le temps des actes. Cette intensification des dispositifs médicaux est observable historiquement, à travers la substitution de technologies à l'approche clinique des corps ; il en va ainsi du recours

\footnotetext{
${ }^{39}$ Art. L2214-2 du Code de la Santé publique : «En aucun cas l'interruption volontaire de grossesse ne doit constituer un moyen de régulation des naissances. »

${ }^{40}$ Ce que souligne l'obtention tardive du remboursement total de l'acte et des examens associés ; décret mis en application le 31 mars 2013.

${ }^{41}$ Pavard (B.), Rochefort (F.), Zancarini-Fournel (M.), Les lois Veil. Les événements fondateurs. Contraception 1974, IVG 1975, Paris, Armand Colin, 2012.
} 
quasi systématique à l'échographie pour le diagnostic ainsi qu'à l'anesthésie lors d'une aspiration.

Dans une structure hospitalière parisienne, nous avons suivi le parcours d'une femme de 29 ans pour un avortement «chirurgical » sous anesthésie générale en 2013. Étalé sur une période de quatre semaines, l'avortement se décompose en de multiples étapes. Ce circuit institutionnel s'ouvre par une visite au Planning familial (examen discursif et mise à disposition d'informations), suivie d'une consultation en laboratoire d'analyses médicales (échodatation de grossesse et prise de sang). Puis, durant une journée à l'hôpital - entre $9 \mathrm{~h} 30$ et $17 \mathrm{~h}$ - se tiennent un rendez-vous « préIVG » avec un gynécologue, une rencontre avec la conseillère conjugale et une consultation avec un anesthésiste. Quatre jours après, une nouvelle journée complète est consacrée, cette fois à la réalisation de l'avortement, durant l'hospitalisation proprement dite. L'intervention ne dure que quelques minutes; le plus long est l'attente ultérieure, le suivi d'intervention codifié depuis 1994. Trois semaines après, a lieu la visite "post-IVG» chez une gynécologue. Son avortement correspond donc à environ deux journées et demie, réparties en quatre lieux, incluant cinq mises à nu (des parties construites comme les plus intimes) face à cinq personnes différentes, et quatre pénétrations vaginales (sonde échographique recouverte d'un préservatif; spéculum et toucher vaginal chez le gynécologue; spéculum, pinces et canules le jour de l'intervention; spéculum lors de la visite de contrôle). La nécessité de l'accès répété à l'intime, l'évidence de son exploration médicale standardisée, ne sont guère questionnées.

Cet enchaînement ordonné des phases, également observé au sein d'un service hospitalier enquêté (hors Paris), constitue une sorte de parcours-type de l'avortement par aspiration avec anesthésie générale en France - auquel il conviendrait d'ajouter les nombreuses pièces administratives et formulaires demandés, les délais à respecter, sans oublier le coût financier des absences au travail qu'endossent les femmes. Ce dispositif d'encadrement médicalisé, particulièrement dense, les confronte à « un ensemble de contraintes d'ordre pratique qui compliquent leurs démarches $»^{42}$ et génère une angoisse. Il les oblige à court terme à demeurer plusieurs heures d'affilée dans des espaces médicaux, et à moyen terme à maintenir une disponibilité de corps et d'esprit pendant plusieurs semaines, ou bien sur un temps plus restreint, mais en des lieux éclatés :

M (24 ans, future avocate, avortement médicamenteux) : « Dans la journée, je devais avoir rendez-vous vers $9 \mathrm{~h}$. À $11 \mathrm{~h}$, j'étais déjà en train de faire la prise de sang, et pour l'écho, les deux centres étaient à côté, donc j'ai vraiment fait comme un marathon, pour retourner chez ma gynéco avec le tout. »

\footnotetext{
${ }^{42}$ Divay (S.), « L'IVG : un droit concédé encore à conquérir », art. cit., p. 217.
} 
De surcroît, on remarque un nombre important d'actes, instruments et regards de diverses professionnelles du domaine sanitaire et social. Toutes ces interventions instaurent un dévoilement verbal relativement poussé de l'intime; autrefois prévu dans la séquence unique de l'entretien psychosocial, il s'est probablement dispersé, et donc multiplié, dans les diverses interactions aujourd'hui nécessaires (médecin et/ou sage-femme, infirmière, échographiste, anesthésiste, intervenante sociale en Planning). Par ailleurs, ces mises à nu et intrusions physiques et discursives étant appelées à se répéter fréquemment en peu de temps, il s'agit en somme d'un condensé de dispositifs gynécologiques qui attend les avortantes.

Notons que certains centres, tel un de ceux enquêtés, ne se contentent pas de proposer l'entretien psycho-social comme prévu par la loi, mais bien de l'inclure d'emblée dans un protocole, renchérissant ainsi les dispositifs étatiques. Au nom du bien-être des femmes, l'institution les tient temporairement, afin de stabiliser leur corps comme lieu d'intervention médicale et de suivi de la sexualité des populations ; car les avortements, très encadrés, peuvent offrir des bénéfices sanitaires importants, dont celui de socialiser au suivi gynécologique.

Actuellement en France sont employées deux méthodes pour avorter, dont l'encadrement est distinct : l'avortement par aspiration et l'avortement par médication. Le premier, dit chirurgical, est ainsi assimilable à un acte lourd et éprouvant, telle une intervention nécessitant une incision. Depuis 2001, l'avortement sur demande de la femme doit être réalisé dans un délai de sept semaines d'aménorrhée s'il l'est par médication, et de sept à quatorze quand il est pratiqué par aspiration ${ }^{43}$. Cette régulation ne s'opère pas sans différenciation sociale, et la manière dont le "choix $»^{44}$ de la méthode se combine aux catégorisations sociales reste encore peu documenté. On sait toutefois que l'avortement par médication (plus d'un avortement sur deux aujourd'hui en France), qui permet à certaines usagères de contourner dans une certaine mesure l'hospitalisation, concerne plutôt «des femmes en couple avec enfants, mais sans IVG précédentes » et " des femmes jeunes et diplômées $»^{45}$, c'est-à-dire des femmes ayant rejoint la norme du «faire famille», ou détentrices d'un certain capital culturel les rapprochant en quelque sorte des praticiennes qui les encadrent. Si cette méthode allège les interactions médicales, elle participe d'un déploiement des interventions médicales à domicile (via les technologies médicamenteuses), transgressant la division domestique/public. Le déplacement spatial des techniques

\footnotetext{
${ }^{43}$ Pheterson (G.), "Avortement pharmacologique ou chirurgical : les critères sociaux du "choix" ", Cahiers du Genre, 31,2001.

${ }^{44}$ Peu de services d'IVG offrent en réalité un choix entre un avortement: par aspiration avec anesthésie locale, par aspiration avec anesthésie générale, ou par voie médicamenteuse.

${ }^{45}$ Vilain (A.), « Les femmes ayant recours à l'IVG : diversité des profils des femmes et des modalités de prise en charge », Revue française des affaires sociales, 1 (1), 2011, p. 116.
} 
institutionnelles et la transformation des actes ne modifient pas pour autant les caractéristiques de l'avortement en milieu médical: de même qu'elles sont 《seules pendant pratiquement toute la procédure hospitalière ${ }^{46}$, les entretiens avec des femmes ayant avorté par médication montrent que l'isolement des femmes - et son corollaire, l'absence des hommes - se reproduisent dans la sphère privée où l'acte d'avortement est plus aisément invisibilisé et euphémisé.

Le champ médico-social sur lequel repose la prise en charge étatique des avortements reconduit le repérage et la responsabilisation de la seule classe des femmes dans la régulation des conduites sexuelles et procréatives. L'accueil et le traitement matériel des avortements traduisent et éternisent l'asymétrie fondatrice qui sous-tend l'organisation sociale du travail re.productif, et ce au-delà de l'hétérogénéité qui caractérise la classe des femmes. La cohérence de cet encadrement sanitaire avec l'ordre patriarcal se poursuit par une prise en charge institutionnelle différenciée selon les groupes de femmes qui avortent.

\section{Des entrepreneuses de morale, garantes des « bons usages » des modes de contrôle des fertilités}

Ce parcours complexe amorce un processus de (ré)éducation aux conduites légitimes en matière de sexualité, auquel les médecins semblent pleinement souscrire. L'application des procédures réglementaires par ces agentes est d'autant plus zélée qu'elles sont pensées comme des exigences minimales. L'un des seuls aspects pour lesquels nous avons observé des stratégies de contournement de la norme légale dans le sens d'un allègement, par plusieurs médecins concerne le délai de réflexion d'une semaine entre le premier rendez-vous et l'acte. Cette volonté de réduire l'attente des avortantes montre que les praticiennes observées excluent tout rôle dissuasif. L'encadrement consiste plutôt à enseigner le devoir contraceptif, alors crédité d'un potentiel effet, celui d'« éradiquer » l'avortement.

La conduite-repoussoir que constitue l'avortement se traduit par des discours réprouvant sa banalisation, grande crainte des pouvoirs publics ${ }^{47}$. Ce message est en grande partie intégré par le corps social ${ }^{48}$, dont les professionnelles de santé, qui, par leur position cruciale, sont chargées de préserver les frontières définies pour ce service public, et de faire en sorte qu'il ne soit pas "détourné» dans ses usages. Une gynécologue médicale (56 ans, installée en cabinet libéral) s'offusque ainsi de l'insouciance des

\footnotetext{
46 Schnegg (C.), «L'avortement médicamenteux : de la technique à l'expérience. La méthode abortive en question », Nouvelles Questions Féministes, 26 (2), 2007, p. 65.

${ }^{47}$ Mossuz-Lavau (J.), «Le débat sur l'IVG à l'automne $2000 »$, Avortement, droit de choisir et santé, Prochoix, 2001.

${ }^{48} \mathrm{La}$ dénonciation des « avortements de confort/convenance » en est une des expressions les plus communes depuis la légalisation; elle est régulièrement instrumentalisée à des fins politiciennes.
} 
jeunes femmes, qui adopteraient indifféremment contraception ou avortement comme méthode de contrôle des naissances :

«Et c'est même pire que ça, parce qu'on entend même certains discours du type “[...] c'est pas grave si je suis enceinte, j'aurais une IVG". Puisque c'est tellement passé dans les mœurs que c'est presque un mode de contraception. Et là, nous sommes arrivés dans ce que moi $\mathrm{j}$ 'appelle la génération des enfants gâtés. Tellement gâtés qu'ils ont perdu la notion de la responsabilisation vis-à-vis de ce mode de vie. »

Ce discours inquiet sur l'indifférenciation des méthodes, représentatif de la position d'un certain nombre de praticiennes rencontrées, illustre la rigidité de la frontière établie entre avortement et modes de gestion anticipés des fertilités, pour lesquels sont définies des implications antinomiques. Entretenir cette polarisation est central dans le processus de stigmatisation de l'avortement, assimilé à la marque d'un mode de vie irresponsable. Selon une logique relationnelle, l'avortement est alors réduit à un échec, une erreur sur la voie de la maîtrise raisonnée de la vie sexuelle. Bien qu'il soit en réalité non réductible à " l'échec contraceptif $~^{49}$, les professionnelles rencontrées n'ont de cesse de déplorer les taux d'avortements, qui devraient selon elles diminuer par le biais d'une "meilleure » médicalisation de la contraception féminine. En outre, la valorisation de cette dernière s'appuie sur des considérations peu en prise avec les conditions matérielles de vie : elle est appréhendée comme méthode facilement accessible, simple d'usage et adaptée à toutes les femmes, qu'il importe de mettre en œuvre en fonction de normes d'efficacité théorique. Ces représentations portées par une partie des praticiennes dévoilent l'occultation des savoirs sociologiques dans la formation médicale ${ }^{50}$. La stigmatisation des avortements s'est donc déplacée: les avortantes, autrefois taxées de «meurtrières», sont aujourd'hui, notamment du fait de la généralisation et de la diversification (théorique) des méthodes de contraception dites efficaces, perçues comme réticentes aux normes de santé ${ }^{51}$, voire irresponsables.

Le continuum de contrôle des fertilités - pouvant s'étendre de l'abstinence à l'infanticide ${ }^{52}$ - est traduit par le corps médical en un système hiérarchisé, en échelle de légitimité établie selon des critères normatifs, définis en situation, où chaque méthode correspondrait à un degré de responsabilité et

\footnotetext{
${ }^{49}$ Bajos (N.), Ferrand (M.), équipe Gine, De la contraception à l'avortement. Sociologie des grossesses non prévues, Paris, INSERM, 2002.

${ }_{50}$ Gelly (M.), Avortement et contraception dans les études médicales. Une formation inadaptée, Paris, L'Harmattan, 2006.

${ }^{51}$ Bajos (N.), Ferrand (M.), "L'interruption volontaire de grossesse et la recomposition de la norme procréative », Sociétés contemporaines, 61 (1), 2006.

${ }_{52}$ Mathieu (N.-C.), « Paternité biologique, maternité sociale... », art. cit.; Tabet (P.), La Construction sociale de l'inégalité des sexes. Des outils et des corps, Paris-Montréal, L'Harmattan, 1998; Tillier (A.), Des criminelles au village. Femmes infanticides en Bretagne (1825-1865), Rennes, Presses universitaires de Rennes, 2001.
} 
de sérieux des femmes. Schématiquement, la contraception d'urgence en occupe le centre. Comme le souligne Gail Pheterson, «présenter l'avortement comme un acte regrettable, le signe d'un manque de jugement ou de discipline, et la contraception comme une démarche éclairée et responsable renforce le mythe selon lequel la contraception ne présenterait aucun risque et l'avortement, même pratiqué dans de bonnes conditions, serait dangereux $»^{53}$.

La stigmatisation qui s'exerce auprès des avortantes se double d'une volonté de les discipliner, de les éduquer aux pratiques légitimes en matière de santé reproductive - l'orthogénie signifiant d'ailleurs littéralement «le redressement/ la régulation de l'engendrement ». Au cours de cet épisode, elles sont soumises à une rengaine entendue dans ces établissements : "il faut qu'on soit sûre que vous ne reveniez pas faire une IVG ». D'abord, l'interaction médicale qui précède l'avortement, tout comme l'ordonnancement de celle-ci, révèle son objectif premier : (ré-)introduire les femmes dans un parcours contraceptif normé. La priorité donnée à la vigilance contraceptive durant les consultations pré-IVG se traduit par le questionnement de la contraception future bien souvent en amont de la présentation des modalités d'avortement, ce qui est significatif d'un contrat tacite: l'acceptation de la contraception médicalisée est une condition d'accès à l'avortement.

Des exigences sont donc implicitement formulées à l'égard des avortantes : 1'adhésion aux normes contraceptives a d'autant plus de chance d'advenir dans cet échange asymétrique, où elles sont en situation de dépendance corporelle face à une autorité médicale. Elle est observable à l'échelle de certains parcours d'avortantes par cette infirmière :

$P$ : « De toute façon, c'est le service où j'ai vu les plus charmantes de toute ma vie. Parce qu'elles sont déjà dans une détresse. Y'a tellement un jugement de valeur par rapport à l'IVG, que quand elles viennent elles ont peur de se faire envoyer sur les roses. Et parfois, on a des super surprises. Moi, $j$ 'avais les filles de l'hôpital de jour qui me racontaient, je l'ai vu de mes propres yeux une fois : une fille adorable, mais charmante, mais vraiment tout le monde, adorable, à l'écoute, qui pose des questions, enfin on avait l'impression qu'il y avait une vraie relation d'échange. Elle va pour faire son avortement, et je la croise dans le hall en train de parler à sa copine, mais de façon odieuse, mais odieuse ! Et pareil avec les infirmières de l'hôpital de jour. Quand elles avaient fini leur IVG, souvent certaines, elles étaient là : "Bon, quand est-ce que je sors? Je comprends pas, c'est rien comme intervention, et puis... je dois attendre l'anesthésiste et tout ça!". C'est pas les mêmes. Elles avaient été adorables, de chez adorable... Après, peut-être que c'est une agressivité qu'elles ont envie de tourner vers elles-mêmes, et

\footnotetext{
${ }_{53}^{5}$ Pheterson (G.), « Grossesse et prostitution. Les femmes sous la tutelle de l'État», Raisons politiques, 3
} (11), 2003, p. 111. 
qu'elles y arrivent pas parce qu'elles ont de la culpabilité, j'en sais rien. Souvent, elle est pas dirigée contre toi l'agressivité en tant que soignant... Elle reflète quelque part une espèce de mal-être, et elle se retourne contre toi, parce que t'es là, au mauvais endroit au mauvais moment. »

Cette observation rend compte de la contrainte temporaire qui pèse sur les avortantes, amenées à se comporter en «patientes-modèles » jusqu'à l'obtention de leur avortement. L'obligation d'allégeance fait parfois partie des stratégies discursives des praticiennes face à des patientes réfractaires :

B (59 ans, gynécologue en libéral, vacations à l'hôpital) : « Mais c'est déjà arrivé qu'elles disent : “Écoutez, je m'en occupe. Oui, j'sais, vous voulez me donner la pilule, moi je ne veux pas la prendre, je me débrouille". Et je leur dis: "Alors si vous vous débrouillez, le jour où vous êtes de nouveau enceinte, et ben vous ne venez pas ici. Nous on ne se débrouillera plus pour vous". J'dis : "C'est pas un plaisir de faire des IVG" [...] Évidemment, dans ce cas-là, autant te dire que la consultation se passe de manière assez agressive. Mais moi je leur dis, et le note dans le dossier: "Refus de la contraception. Prévenue que si de nouveau enceinte, on ne refera plus l'IVG dans le service". Ce qui est faux hein. »

Ces menaces de non-prise en charge, même mensongères, sont considérées par les enquêtées comme des outils utiles au redressement des comportements réputés hors normes. Tout en soulignant la déviance, la menace d'un refus d'avortement dans le cas d'une nouvelle grossesse non souhaitée obéit à une logique coercitive - la suppression d'un droit - en même temps qu'elle promeut l'intégration de la norme contraceptive, modèle consacré et remède.

Différentes rhétoriques jalonnent le processus de normalisation de ces femmes faisant preuve d'une gestion de leur fertilité échappant à la raison médicale. L'une d'entre elles, certainement puissante tant elle existe au-delà des limites d'exercice du médical, consiste à jouer insidieusement sur les légendes noires de l'avortement. Une célèbre formule permet de relayer le discours consensuel sur le caractère problématique de l'avortement, qu'il convient d'endiguer par des procédés de prévention :

A (29 ans, gynécologue hospitalière) : «Je leur parle de l'avortement à ce moment-là. Je leur dis que l'avortement c'est pas anodin: “psychologiquement c'est difficile à vivre, physiquement c'est difficile à vivre, c'est un geste qui n'est pas anodin dans votre utérus". »

S (61 ans, gynécologue en libéral, vacations à l'hôpital), à une patiente de 17 ans, consultation pré-IVG : «Vous ne trouvez pas ça naturel la pilule, c'est ça ? Ben parce que ce qui compte pour nous, c'est que vous ne reveniez pas faire une IVG. Parce qu'une IVG, ce n'est pas un geste anodin. »

Le recours à cette antienne par le corps médical reconduit des 
représentations idéologiques consistant à associer les conduites stigmatisées à une échelle de risque élevée, et vice-versa ${ }^{54}$. Ce discours a beau être contraire à la précision censée diriger tout avis médical, il permet à certaines professionnelles de soin de se représenter de manière systématique, faute de consensus scientifique, la gravité de ce moyen de contrôle des fertilités, tout en investissant la déviance d'une caution savante. Ensuite, elles peuvent trouver un intérêt à faire appel à ce langage familier, car il les dispense de préciser les conséquences supposées d'un avortement (troubles psychologiques, dommages sur l'utérus). Le rappel régulier du «non anodin » suggère subrepticement son caractère nocif et sinistre sans pourtant pouvoir le justifier; il est une façon d'entretenir l'ambivalence et de susciter l'anxiété, le malaise vis-à-vis de ce mode de maîtrise procréative. L'observation des interactions montre que les médecins démentent peu ce type de discours. À une avortante de 35 ans ayant expliqué l'origine de ses réticences envers «les hormones", D (24 ans, interne en gynécologie médicale) renverse les rapports générationnels en répliquant : «Ah non, mais là il faut une contraception. Parce que votre mère elle aura beau vous dire que la pilule c'est pas bon pour la santé, mais une IVG ce n'est pas bon non plus ». Ces commentaires péremptoires, parfois infantilisants, diffusent la représentation d'un geste invasif et violent sur les corps, pouvant entamer une fertilité future. Ils remplissent une fonction dans l'encadrement des avortantes, celle de (ré-)engager un parcours contraceptif impliquant un suivi gynécologique, lequel est associé à l'idée d'un gain d'autonomie et de bienêtre pour les femmes.

Les discours des agentes institutionnelles confortent le jugement social de déviance qui s'abat sur les avortantes, autrement dit participent d'une pression sociale diffuse. «La situation d'IVG c'est une situation de détresse, quoi qu'on en dise », martèlent ainsi des médecins et conseillères conjugales. Malgré la suppression récente dans la loi de la « situation de détresse ${ }^{55}$ qui conditionnait l'accès à l'avortement à des états psycho-sociaux exceptionnels, perdurent en fait dans la culture professionnelle de celles qui la mettent en œuvre des représentations disqualifiantes de l'avortement, vu comme épisode difficile, voire traumatisant pour les corps et esprits.

De même, au nom de la régulation légitime de la procréation, et donc de l'ordre social, les praticiennes - y compris les plus réticentes à l'application $\mathrm{du}$ dispositif de recueil de la parole - sont conduites à faire parler les usagères, en amont de l'avortement. La référence récurrente au bien-être mental, l'incitation (parfois l'obligation) à rencontrer la conseillère conjugale $\mathrm{du}$ service, opèrent une psychologisation de ce parcours ${ }^{56}$. Constat que

\footnotetext{
${ }^{54}$ Pheterson (G.), « Grossesse et prostitution. Les femmes sous la tutelle de l'État », art. cit., p. 111.

${ }^{55}$ Vote de l'article « 5 quinquies $C$ » du projet de loi sur l'égalité femmes-hommes, 21 janvier 2014.

${ }^{56} \mathrm{Ce}$ dès la mise en application de la loi Veil. Devreux (A.-M.), « De la dissuasion à la normalisation. Le rôle des conseillères dans l'entretien pré-IVG », art. cit.
} 
corrobore l'implication forte des psychologues - assurant 39,8\% des entretiens psycho-sociaux (DREES, 2009) - dans cette séquence de l'avortement. L'articulation des rendez-vous entre médecins et conseillère de planification familiale, ainsi que l'observation d'interactions, portent à formuler l'hypothèse d'un travail de repérage des femmes auprès desquelles il convient de mobiliser les affects. La fertilité et la maternité sont au cœur des lectures médicales qui ont parfois pour visée explicite de déchiffrer l'inconscient, du moins certains signifiants dans le discours des sujets, mais aussi de «faire pleurer». M (29 ans, deux avortements) raconte ainsi en entretien une interaction avec une médecin se terminant par des larmes, alors même qu'elle se sentait à l'aise avec sa décision initiale; l'attitude de la praticienne avait consisté à orienter l'échange verbal vers la «contradiction » entre le non-désir d'enfant de $\mathrm{M}$, alors en couple depuis deux ans, et son absence de contraception médicalisée, suggérant par là un désir de grossesse ou d'enfant inconscient. Ici, le repérage de l'avortante à encadrer psychologiquement vise une femme réunissant les conditions sociales légitimes pour procréer ${ }^{57}$ (couple et emploi «stables», proche de la trentaine).

Lors d'une consultation avec une femme de 36 ans, un conseiller conjugal analysait son épisode d'avortement - interprétation qu'il nous confirma en aparté - comme l'activation d'une expérience problématique avec ses aïeuls, et ponctuait l'entretien d'incitations à l'expression des émotions : «J'entends aussi de la colère de votre part», «laissez libérer vos émotions », disait-il quelques secondes avant la crise de sanglots de l'avortante. Ceux-ci semblent identifiés par les professionnelles moins comme le produit d'une culpabilisation - parfois ressentie comme telle par des patientes, ainsi que le confie $\mathrm{M}$ en entretien - que comme un élément cathartique, quasi curatif psychologiquement. Cette conceptualisation de l'avortement est loin d'être l'apanage du corps médical. Ce qui est toutefois remarquable, c'est l'usage professionnel qui en est fait, lorsque la création ou l'entretien d'émotions s'apparente à un outil de travail. Il consiste notamment à valoriser les larmes, c'est-à-dire la tristesse qu'il convient d'éprouver, probablement en soupçonnant, derrière leur absence, un déni des affects potentiellement nocif. Par ce gouvernement des subjectivités, les professionnelles encadrant les parcours d'avortement relayent un modèle du vécu de l'avortement, impliquant que ses ressorts physiologiques sont inséparables de troubles psychologiques - raisonnement au demeurant en vogue auprès de certaines médecins proches du courant de la "gynécologie psychosomatique». Le registre émotionnel peut également être considéré comme une « voie royale » pour accéder aux cadres cognitifs, les influencer. Cette surveillance discursive, de pair avec le contrôle corporel, compte parmi

\footnotetext{
${ }^{57}$ Macintyre (S.), « Qui veut des enfants ?», Nouvelles Questions Féministes, 3, 1978.
} 
les dispositifs pratiques qui dramatisent cet épisode autant qu'ils le transforment en levier de normalisation des comportements.

Parmi les prescriptions sociales dont le monde médical se fait le relais, figurent les temporalités procréatives et, avec elles, les normes contraceptives, en tant que technologies appliquées aux corps féminins et conjurant le risque d'avortement. Dans un contexte où "l'autocontrôle légitime de la reproduction relève $[. .$.$] des projets à moyen terme plutôt que$ des réactions de court terme $\gg{ }^{58}$, il convient d'appréhender la contribution de la contraception à la médecine préventive en tant que mode de contrôle social. L'encadrement étatique des avortements que mettent en œuvre les agents du secteur médical et social consiste à domestiquer les comportements et affects afin de les rendre conformes à une gestion légitime des activités sexuelles - en l'occurrence, une femme ne souhaitant pas d'enfant est sommée de conserver en continu sa sexualité sous contrôle médicamenteux. Dans les établissements enquêtés, l'implant contraceptif ou le stérilet est posé après l'acte d'avortement, au bloc; la visée explicite de cette politique - maintenue malgré les nombreux cas de rejet de stérilet dans les jours suivants $^{59}$ - est de fournir des corps sous protection contraceptive immédiate. La consultation dite post-IVG complète ce parcours institutionnalisé d'apprentissage: cette dernière étape, régulièrement escamotée par les patientes, est promue comme phase de vérification de leur observance contraceptive.

Au cours d'une interaction avec G (67 ans, gynécologue, libérale et hospitalière), une femme de 30 ans (dont l'hexis corporel laisse supposer qu'elle est issue des classes populaires), trois semaines après l'IVG, dit faire « une grosse déprime» et regrette d'avoir avorté. Elle est persuadée que la pose de l'implant contraceptif, réalisée le jour même de l'intervention, participe de son mal-être, et en exige donc le retrait. La gynécologue la suspecte de "vouloir repasser à la pilule pour pouvoir [se] donner les moyens de l'oublier » et n'interroge pas les éventuels effets secondaires de l'implant. Réticente, elle opte pour un règlement de ses maux par la parole, c'est-à-dire la rencontre du psychologue du service d'IVG. Ces repérages et étiquetage d'une vulnérabilité - la nécessité d'éclaircir son rapport à un éventuel "désir d'enfant»-s'avèrent propices à la normalisation du comportement contraceptif. Dans ce cas précis, la médecin refuse de répondre à la requête de la patiente, et lui impose un délai de réflexion en « inventant» une convocation de retrait d'implant. Sa priorité étant de ne pas désavouer l'option contraceptive, elle tient à garder cette patiente jugée instable sous mainmise thérapeutique (contraception hormonale « en

\footnotetext{
${ }^{58}$ Gelly (M.), Avortement et contraception dans les études médicales. Une formation inadaptée, Paris, L'Harmattan, 2006, p. 171.

${ }^{59}$ Rejet dû à une pose de stérilet sur un col d'utérus ouvert après l'IVG.
} 
continu ») et préconise un suivi psychologique. En déniant à l'usagère une part de son autonomie décisionnelle, la gynécologue tient un rôle d'agente morale $« \mathrm{p}(/ \mathrm{m})$ aternaliste $»$ et, en raisonnant dans un cadre plus global, de contrôle social.

Cette situation d'imposition de contraception n'est qu'un exemple parmi d'autres de la fonction de domestication attribuée aux professionnelles de soin dans les parcours des avortantes, rôle qu'elles investissent à leur tour d'un sens positif et salvateur. En amont comme en aval de l'acte, l'objectif est bien de maintenir une vigilance sur des comportements inhabituels, de susciter l'adhésion au modèle biomédical de maîtrise des corps. On observe alors que, sous les atours de la neutralité scientifique et du savoir médical, s'exerce un encadrement normatif de la classe des femmes. Pour analyser finement les déclinaisons du contrôle effectué auprès des femmes, il conviendrait d'inclure l'encadrement particulier réservé à certaines catégories sociales, dont font probablement partie les femmes racisées et des classes populaires. Toutefois, nous choisissons de nous concentrer sur des facteurs plus méconnus, particuliers à cette prise en charge médicale, et qui trouvent leur place dans l'idéologie patriarcale.

\section{Des expertes porteuses d'une échelle de la déviance}

À l'instar du système carcéral et pénal, « l'agencement de ces dispositifs [de contrôle social] prend des formes différentes en fonction de leur degré de conformité ou de déviance aux rôles sexués ${ }^{60}$. La stigmatisation des avortantes dans l'enceinte médicale comporte des gradations. Parmi elles, on note le nombre ainsi que la proximité des avortements, mais aussi le nombre de semaines associé au fœtus. Il convient de garder à l'esprit que ces « degrés de stigmatisation » sont sans doute modulés par la perception des propriétés sociales des usagères, dont leur âge, leur situation matrimoniale et familiale. Car la vigilance médicale est d'autant plus forte qu'elle porte sur un « certain » type de femmes. Hélène Bretin l'a démontré dans ses travaux sur la prescription des injectables comme instrument de contrôle social des minorités étrangères ${ }^{61}$. Nos observations et les entretiens menés amènent à formuler 1'hypothèse que les implants contraceptifs et les stérilets jouent actuellement ce rôle auprès de certains groupes : des femmes avortant en suites de couches, celles en situation de très grande précarité avec plusieurs enfants, des femmes étrangères, des femmes de moins de 20 ans issues des classes populaires, et plus généralement celles suspectées de ne pas être capables d'évaluation rationnelle du comportement.

S (65 ans, gynécologue en libéral) : « Après y’a les récidivistes de l'IVG. Celles-là on leur fera jamais prendre de pilule, hein. Y'a toujours la solution

\footnotetext{
${ }^{60}$ Cardi (C.), « Le contrôle social réservé aux femmes : entre prison, justice et travail social », art. cit., p. 19.

${ }^{61}$ Bretin (H.), Contraception, quel choix pour quelle vie? Récits de femmes, paroles de médecins, Paris, INSERM, 1992.
} 
de l'IVG, et c'est tout. Clairement, c'est tout, hein. [...] tous les médecins vous le diront. »

Le terme "récidiviste », entendu à plusieurs reprises dans les structures observées, désigne les femmes transgressant radicalement le modèle acceptable de vie sexuelle et reproductive. Plus qu'une pathologisation, cette expression issue du champ lexical pénal (qui pourrait lui préférer «réitération d'infraction») les étiquettes explicitement comme des coupables, qui prendraient une habitude nuisible. Ce terme désignant «la répétition d'un acte criminel » a ceci d'étonnant qu' « il traduit un consensus qui unit défenseurs et adversaires de l'avortement: celui-ci ne saurait être toléré s'il se répète $»^{62}$. Ces avortantes échappent pour partie à la stratégie de domestication sanitaire des comportements des femmes, dans la mesure où, malgré les processus de "rattrapage» amorcés lors de chaque prise en charge médicale des avortements, elles y ont recours plusieurs fois dans leur vie.

Les représentations de cet usage de l'avortement, ancrées dans des considérations d'ordre moral, oscillent entre culpabilisation ${ }^{63}$ et psychologisation $^{64}$ des femmes. Les usagères concernées représentent aux yeux des professionnelles encadrant ces parcours «l'exception parmi les femmes normales qui demandent» l'avortement; elles auraient «une personnalité déjà désadaptée, $[\ldots]$ un comportement pathologique $»^{65}$. Les commentaires relevés dans les services orientent vers une interprétation pathologisante et/ou victimisante de ces comportements marginaux de régulation des naissances, où les femmes sont volontiers décrites comme égarées ou immatures.

L'interprétation des avortements répétés atteint une échelle plus élevée de déviance lorsqu'ils surviennent de façon rapprochée. Plus la durée entre les avortements est courte, plus les jugements moraux sont explicites: les stigmates de l'irrationalité et l'irresponsabilité, de la marginalité psychosociale, voire de la maladie mentale se font jour. Car ces avortements itératifs ${ }^{66}$ incarnent la «banalisation de l'avortement», péril qui oriente prioritairement ce domaine de l'action publique.

Eliot Freidson identifie chez le médecin «entrepreneur moral» une inclination à la «surdéclaration » de maladies, c'est-à-dire « la création par

\footnotetext{
${ }^{62}$ Novaes (S.), « Les récidivistes », Revue française de sociologie, 23 (3), 1982, p. 473.

${ }^{63}$ Bajos (N.), Ferrand (M.), « De l'interdiction au contrôle : les enjeux contemporains de la légalisation de l'avortement », Revue française des affaires sociales, 1 (1), 2011, p. 52.

${ }^{64}$ Elle peut prendre des formes diverses, comme en témoignent les tentatives de formalisation du «Syndrome post-avortement » aux États-Unis et en Grande-Bretagne. Cf. Lee (E. J.), Abortion, Motherhood and Mental Health: The Medicalization of Reproduction in the U.S. and Britain, New York, Aldine Transaction, 2003.

${ }^{65}$ Novaes (S.), « Les récidivistes », art. cit., p. 475.

${ }^{66}$ « Soit la femme a eu 3 ou plus de 3 IVG, soit elle a eu une $2^{\text {e }}$ IVG à moins de 3 ans d'intervalle. » Garel (M.), Crost (M.), Kaminski (M.), « Caractéristiques psychologiques et sociales des femmes qui ont des IVG itératives. Enquête dans trois centres français », Contraception, Fertility, Sexuality, 24, 1996, p. 2.
} 
la profession d'une image de la maladie plus répandue qu'elle ne l'est en réalité $\rangle^{67}$. De manière similaire, ces situations de recours pluriels à l'avortement sont érigées par le corps médical en figures « extrêmes » de la déviance féminine. En référence à la «sur-criminalisation» qu'identifie Coline Cardi à propos des comportements de prisonnières mettant en jeu le statut maternel, on pourrait parler de « sur-stigmatisation/psychologisation » des pluri-avortantes, d'autant plus si l'acte est répété sur un temps court. Ces situations ont beau être minoritaires quantitativement, tant la normalisation contemporaine des conduites reproductives est opératoire auprès des femmes, les professionnelles les surestiment ${ }^{68}$. Elles sont ensuite instrumentalisées dans les discours en tant que cas d'école d'usages imprudents de l'avortement: la référence à ces conduites fautives, dès lors répréhensibles, sert à ériger une nouvelle frontière, au sein même du groupe étiqueté comme déviant, entre les avortantes ponctuelles et les avortantes chroniques («à répétition », disent usuellement les médecins) - au sens où leur mode de régulation des fertilités est dit pathologique. Les femmes présentant leur demande d'avortement au-delà des délais autorisés incarnent également un degré très élevé de déviance.

$\mathrm{Au}$ sein du continuum des méthodes de régulation des naissances, se dessine une sorte de classement des avortantes, avec une gradation latente des déviances : l'avortement précoce (à un stade moindre d'avancée de la grossesse) et légitime - c'est-à-dire d'une part lorsque les conditions socialement requises pour être mère ne sont pas réunies, et d'autre part lorsque cet avortement survient dans une situation d'adhésion aux normes contraceptives - défini comme pratique acceptable au sein même de la gestion de la déviance, occupe un pôle ; à l'opposé, on trouve l'avortement itératif, tardif et illégitime - lorsque les conditions socio-économiques de la maternité sont réunies -, dont l'encadrement entraîne une condamnation morale et une pathologisation. Ces usagères sont visées par l'institution médicale comme population-cible à surveiller de près, sur lesquelles exercer un contrôle plus étroit par le biais de prescriptions performantes qui, parfois, s'apparentent à une coercition contraceptive ${ }^{69}$. Ce ciblage, et la logique de prédiction qui l'accompagne - toute avortante est une potentielle récidiviste -, fait partie des mécanismes qui stigmatisent les avortements en général, et, dès lors, condamnent toutes les femmes, avortantes en puissance.

\footnotetext{
${ }^{67}$ Freidson (E.), La profession médicale, Paris, Payot, 1984 [1 $1^{\text {re }}$ éd. am. 1970], p. 275.

${ }^{68}$ Cardi (C.), «Le contrôle social réservé aux femmes : entre prison, justice et travail social », art. cit.

${ }^{69} \mathrm{Cf}$. Bretin(H.), Contraception, quel choix pour quelle vie ? Récits de femmes, paroles de médecins, op. cit.
} 


\section{Derrière la reconfiguration du stigmate, la pérennisation du contrôle des femmes}

Nos enquêtes font apparaître des agentes majoritairement acquises au principe selon lequel les avortements sont un trouble à l'ordre sexuel et moral, ou du moins " un problème », un événement à éviter. Bien entendu, les catégories d'entendement et pratiques des médecins sont fonction de leurs dispositions personnelles, ainsi que d'un éthos professionnel qui résulte notamment de leur rapport à l'institution, du type de socialisation professionnelle, et d'une éventuelle expérience militante. Sans pour autant ignorer ces différences, nos observations montrent qu'elles agissent comme gardiennes d'une partie de l'ordre sexuel, en usant de pratiques pédagogiques qui, bien qu'elles aient évolué avec les modifications législatives, se nourrissent de présupposés communs. En cela, les anciennes militantes de la «maternité choisie» ne se soustraient qu'en partie aux représentations dominantes de l'avortement $^{70}$ et de la vulnérabilité particulière des femmes, qui continuent de traverser le corps social.

De toute évidence, la diversité des pratiques médicales est accrue par l'interaction avec des femmes d'âges et d'origines sociales variés puisqu'aucun groupe de femmes "en âge de procréer» ne semble faire exception dans l'expérience de l'avortement. Pour autant, les mécanismes généraux de surveillance, les marges de négociation des usagères, mais aussi les croyances des agentes, révèlent une communauté de traitement des avortantes. Ces dispositifs participent à la constitution d'un groupe homogène, la classe de sexe femmes, dont le corps est construit comme objet de gouvernement, censé rejoindre les modèles prescrits en matière de maternité et de maîtrise des fertilités.

L'implication des hommes dans la contraception des partenariats et la charge mentale qu'elle représente n'est pas envisagée dans ces parcours. Par ailleurs, l'avortement est assorti d'un éventail de gestes et discours intégralement orienté autour du coït hétérosexuel, de fait défini comme épuisant le répertoire sexuel ${ }^{71}$, occultant dès lors toute réflexion sur une forme de sexualité indépendante de la santé reproductive. En cela, le contrôle moderne des naissances ne dissocie que partiellement sexualité et procréation. Ces institutions médicales forment donc une sorte de «police de la procréation » qui renvoie les femmes à un usage de leur corps au féminin, dominé par l'impératif maternel, que les patientes ont pour mission d'ordonner à l'occasion des avortements.

\footnotetext{
${ }^{70}$ Caenen (M.), «Avorteuses et avortées ». Étude d'un centre IVG du Nord, mémoire de M1 de science politique, Université Lille 2,2014.

${ }_{71}^{71}$ Tabet (P.), «Fertilité naturelle, reproduction forcée», in La construction sociale de l'inégalité des sexes. Des outils et des corps, Paris, L'Harmattan, 1998 [1 ${ }^{\text {re }}$ éd.1985].
} 
Le contrôle étatique des avortements, tel que délégué aux professionnelles de soin, contribue à la pérennisation des rapports sociaux de sexe en matière de procréation, lesquels s'articulent avec l'ancrage social des comportements hiérarchisés, en termes d'âge, de classe sociale, de race, de sexualité. En ciblant certaines femmes, puis en les encadrant, les domestiquant et les normalisant du point de vue de la régulation de leurs sexualités, l'institution médicale est partie prenante des " étiqueteurs » qui produisent la déviance. On peut dire comme Coline Cardi à propos du système pénal, mais cette fois en considérant le champ médical, que cette surveillance "n'est qu'un instrument de contrôle des femmes parmi d'autres », complémentaires les uns des autres pour les «surcontrôler [...] au quotidien de façon plus ou moins formelle ou institutionnalisée $\gg^{72}$. L'avortement construit comme parcours éprouvant et sombre, culpabilisant les femmes pour avoir failli à leur devoir de maîtrise de leur fertilité, les conduit vers les normes contraceptives. La contraception médicalisée représente quant à elle un formidable relais, sans doute plus subtil et diffus, de maintien d'une emprise politique via les professionnelles de la santé reproductive sur les corps des femmes.

Si les avortantes ne sont plus considérées comme des meurtrières, que la culpabilité est amoindrie, celles qui se soustraient au modèle biomédical de la procréation (qu'elles aient une marge de manœuvre plus étroite en la matière ou qu'elles nourrissent à son égard une suspicion) demeurent traitées comme des irresponsables, fautives et à (re)dresser. "L'analogie statutaire » entre avortante et délinquante conserve toute sa pertinence si on considère " un champ logique [...] déjà parcouru pour l'enfance, dans lequel la faute n'est plus à punir, mais à "comprendre" et à "gérer" à l'aide d'un arsenal conceptuel fourni par d'autres disciplines : psychologie, psychologie sociale, psychiatrie $»^{73}$. Le changement de référentiel suite à la légalisation de l'acte a impliqué la suppression de la punition légale au profit d'un accompagnement individualisé et une éducation par les professionnelles de soins aux «bonnes pratiques » de gestion des fertilités. En tous les cas, les modalités routinières d'exercice de ce traitement n'ont pas évacué la culpabilisation. On assiste plutôt à un renouvellement du stigmate, à travers une "déviance morale légale $\gg^{74}$, qui s'ajoute en quelque sorte à une autre déviance : le corps des femmes et leur étiquetage au sein d'une médecine conçue selon des principes androcentrés.

\footnotetext{
${ }^{72}$ Cardi (C.), « Le contrôle social réservé aux femmes : entre prison, justice et travail social », art. cit., p. 7.

${ }^{73}$ Serverin (E.), «De l'avortement à l'interruption volontaire de grossesse : l'histoire d'une requalification sociale », Déviance et société, 4 (1), 1980.

${ }^{74}$ Divay (S.), « L'IVG : un droit concédé encore à conquérir », art. cit., p. 199.
} 
Marie Mathieu est doctorante en sociologie en cotutelle au Cresppa-CSU, Paris 8 et à l'Institut de Recherches et d'Études Féministes de l'Université du Québec à Montréal, sous la codirection de Michèle Ferrand et Francine Descarries. Après des enseignements à l'Université Paris 8, elle a participé au traitement et à l'analyse de données du Recensement national des recherches sur le genre et/ou les femmes à la Mission pour la place des femmes du CNRS. Son travail de thèse explore la construction sociale de l'épisode de l'avortement en France et au Québec, à partir de récits de femmes ayant arrêté une ou plusieurs grossesses dans ces deux sociétés.

\section{marie.juliette.mathieu@gmail.com}

Lucile Ruault est doctorante en sociologie politique au Centre d'études et de recherches administratives, politiques et sociales (CERAPS) et enseigne à l'Université Lille 2. Elle prépare une thèse, sous la direction de Rémi Lefebvre et Frédérique Matonti, sur les mobilisations de femmes dans le domaine de la santé, pour l'appropriation d'un pouvoir sur leur corps, principalement à partir des groupes revendiquant la contraception et l'avortement libres dans les années 1970 en France - dont les MLAC - et des groupes de self-help. Elle s'intéresse plus généralement à l'encadrement médical des femmes, notamment à travers ses recherches de master 2 sur la spécialité de gynécologie médicale dont cet article présente quelques résultats.

lucile.ruault@yahoo.fr 